\title{
A fauna de abelhas sem ferrão em áreas urbanas: 50 anos de estudos e prioridades de pesquisa no Brasil
}

\author{
The stingless bee fauna of urban areas: 50 years of studies and research \\ priorities in Brazil
}

\author{
S. J. L. dos Santos*; B. C. Barbosa; F. Prezoto \\ Laboratório de Ecologia Comportamental e Bioacústica (LABEC), Programa de Pós-Graduação em Biodiversidade e \\ Conservação da Natureza, Universidade Federal de Juiz de Fora, Juiz de Fora-MG, Brasil
}

*samuel.lima@icb.ufjf.br

(Recebido em 24 de agosto de 2020; aceito em 16 de dezembro de 2020)

\begin{abstract}
As abelhas sem ferrão são fundamentais para a manutenção de áreas verdes presentes em centros urbanos, através da polinização. Contudo, o ambiente urbano oferece uma série de desafios que influenciam a riqueza e diversidade desses insetos. Nós objetivamos realizar uma análise detalhada dos estudos de diversidade de abelhas sem ferrão em áreas urbanas publicados nos últimos 50 anos. Para a revisão de literatura, foram selecionadas as publicações que estavam indexadas em bases de dados conhecidas, com palavras-chave indicando o assunto, dentro do período de tempo requerido para a análise. Foram encontradas 44 publicações, a maioria publicada em revistas científicas $(77,27 \%)$. Os estudos começaram a ser publicados regularmente a partir do começo da década de 2000, sendo 2003, 2005, 2010 e 2012 os anos mais produtivos. Houve concentração de publicação de estudos nos estados do Paraná, Minas Gerais e São Paulo. Entre os estudos foram utilizados seis diferentes métodos de coleta, sendo que, os métodos de Busca Ativa (ou Varredura) e Busca por Ninhos demonstraram ser os mais utilizados. Verificamos que as espécies mais encontradas em ambiente urbano, segundo as publicações, são Trigona spinipes e Tetragonisca angustula. Almejamos com as informações presentes nessa análise contribuir para o conhecimento sobre a diversidade urbana de abelhas sem ferrão, além de indicar locais prioritários para a execução de futuros estudos com o grupo no Brasil.

Palavras-chave: meliponina, diversidade, meta-análise
\end{abstract}

Stingless bees are essential for the maintenance of green areas present in urban centers through pollination activities. However, the urban environment offers a number of challenges that influence the richness and diversity of these insects. We aim to carry out a detailed analysis of studies on the diversity of stingless bees in urban areas published in the last 50 years. For the literature review, they were selected as publications that were indexed in databases, with keywords indicating the subject, within the period of time required for the analysis. We found 44 publications, most of them published in scientific journals $(77.27 \%)$. The studies started to be published regularly from the beginning of the 2000s, and the years 2003, 2005, 2010 and 2012 were the most productive years. There was a concentration of studies in the states of Paraná, Minas Gerais and São Paulo. Among the studies, six different collection methods were used, and the Active Search (or Sweep) and Nest Search methods proved to be the most used. We also discovered, according to the publications, that the most found species in an urban environment are Trigona spinipes and Tetragonisca angustula. We aim with the information present in this analysis to contribute to the current status of knowledge about the urban diversity of stingless bees, in addition to indicating priority places in Brazil for carrying out future studies with the group.

Keywords: meliponina, diversity, meta-analysis

\section{INTRODUÇÃO}

O processo de urbanização provoca mudanças intensas e imprevisíveis que afetam a diversidade de abelhas em biótopos naturais, através da redução da composição florística e alteração dos locais utilizados para nidificação $[1,2,3,4]$. O desenvolvimento de biótopos urbanos, chamadas de áreas verdes, favorece o estabelecimento de algumas espécies, ao passo que dificulta o de outras, podendo provocar sua extinção $[1,3,5,6]$. A preocupação com essas mudanças e seus impactos sobre a fauna de abelhas nativas estimulou a execução de estudos de diversidade pelo Brasil.

Apesar da grande contribuição das expedições realizadas por naturalistas, como Cockerell (1900) [7], Ducke (1902-1910)[8 -12], Friese (1910) [13] e Schrottky (1902) [14] no começo do $128001-1$ 
século 20 para o conhecimento da melissofauna brasileira, foi somente com o estudo pioneiro realizado por Sakagami, Laroca e Moure (1967) [15] que os trabalhos de levantamento de abelhas pelo Brasil passaram a ter um foco biocenótico e metodologia padronizada (busca ativa ou varredura), caracterizada em capturar as abelhas enquanto visitavam as flores em busca de recursos. A partir de então, esse método foi amplamente aplicado e modificado em estudos realizados pelo país que visaram obter informações sobre a biologia e ecologia desses insetos.

A realização de estudos de inventário para a subtribo Meliponina, conforme classificação utilizada por Silveira, Melo e Almeida (2002) [16], é fundamental, devido à sua grande importância ecológica; visto que são responsáveis pela polinização de até $90 \%$ da vegetação nativa e altamente influenciadas por mudanças ocorridas no ambiente, sendo consideradas bons indicadores ambientais $[17,18,19]$. Portanto, as informações recolhidas nesses trabalhos contribuem para aprimorar o conhecimento existente sobre a fauna de meliponíneos brasileiros e permite o direcionamento de projetos de monitoramento, manejo e conservação das espécies.

Algumas espécies generalistas de meliponíneos estão presentes em áreas urbanas, favorecidas pelas condições criadas pelas atividades humanas, como a disponibilidade de recursos alimentares em plantas cultivadas em parques e jardins urbanos e cavidades presentes em construções, que são utilizadas para nidificação [2, 20, 21,22]. A fim de contribuir com informações acerca da fauna de abelhas sem ferrão urbana, o presente estudo visou analisar detalhadamente os estudos de diversidade de meliponíneos em ambiente urbanizado realizados no Brasil nos últimos 50 anos. Além disso, realizamos uma discussão sobre avanços e prioridades de pesquisa sobre o tema no Brasil.

\section{MATERIAL E MÉTODOS}

Para este estudo foram seguidos os passos propostos pela metodologia de PRISMA para estudos sistemáticos e de meta-análise [23] adaptada por Moher et al. (2015) [24]. A abordagem metodológica incluiu o desenvolvimento de critérios de seleção, definição de estratégias de busca, avaliação da qualidade do estudo e extração de dados relevantes.

Os critérios para seleção e inclusão das publicações foram: publicações que tratam do assunto; publicações indexadas nas bases de dados Google Acadêmico, Scientific Electronic Library Online (SciELO), Scopus e Web of Science; artigos publicados em periódicos dentro do período de tempo definido de janeiro de 1967, ano de publicação do primeiro estudo, a dezembro de 2019. As palavras-chave utilizadas para a busca nas bases de dados foram: abelhas sem ferrão, stingless bees, bees, diversidade, diversity, richness, riqueza, urbano, urban enviroment, urban bees. Publicações como monografias, dissertações, teses e livros foram adicionados através de referenciamento cruzado.

De cada publicação foram registradas as seguintes informações: localidade e duração dos estudos, métodos de coleta e número de espécies de abelhas sem ferrão identificadas. Dos trabalhos que utilizaram o método Busca por Ninhos foram retiradas informações acerca do número de ninhos registrados, substratos utilizados para nidificação e altura em relação ao solo. Baseado na coleta dessas informações, foram gerados: (1) um mapa de distribuição dos estudos pelo Brasil; (2) uma tabela das espécies registradas em ambiente urbano, os estados onde foram registradas, os métodos utilizados para coletá-las e informações relativas aos seus ninhos.

O índice de constância das espécies registradas nos estudos através dos métodos utilizados foi calculado pela fórmula: $\mathrm{C}=\mathrm{P} \times 100 / \mathrm{N}$, onde: $\mathrm{P}=$ Número de estudos em que houve registro de determinada espécie e N = Número total de estudos. Segundo Bodemheimer (1955) [25] o resultado pode ser enquadrado nas seguintes categorias: Espécie constante: registrada em mais de $50 \%$ dos trabalhos; Espécie acessória: presente entre $25 \%$ a $50 \%$ dos trabalhos; Espécie acidental: presente em menos de $25 \%$ do total de trabalhos.

Para analisar a riqueza de espécies de abelhas sem ferrão na matriz urbana, foram confeccionadas curvas de rarefação de espécies (senso Gotelli e Colwell, 2001) [26] com o programa EstimateS9 [27] com 5000 aleatorizações. Os estimadores não-paramétricos Jackknife de $1^{\mathrm{a}} \mathrm{e} 2^{\mathrm{a}}$ ordem foram utilizados para projetar a riqueza de espécies máxima que uma matriz urbana poderia alcançar. O programa gera curvas de acumulação de espécies aleatorizando a ordem das 
amostras; assim, cada ponto da curva corresponde à média de riqueza acumulada nas 5000 curvas e está associado a um desvio-padrão. Cada estudo foi considerado uma amostra, o que resultou em 44 amostras.

\section{RESULTADOS E DISCUSSÃO}

Foram encontradas 44 publicações, sendo um livro (2,27\%), nove estudos não publicados $(20,45 \%)$ (monografias, dissertações e teses) e 34 artigos publicados em revistas científicas (77,27\%). Em relação aos artigos científicos, foram utilizadas 25 revistas para suas publicações (Tabela 1). A revista mais utilizada foi a Acta Biológica Paranaense, com cinco trabalhos publicados, seguida por EntomoBrasilis, Biota Neotropica e Bioscience Journal, com dois trabalhos publicados cada, representando assim 25\% $(\mathrm{n}=11)$ dos artigos publicados. Dessas 25 revistas, a maioria $(n=18)$ são periódicos nacionais, corroborando a relevância do tema no cenário nacional.

Tabela 1: Publicações, meio de publicação, área de estudo (estado) duração de estudo, métodos de coleta, e número de espécies registrados em estudos de diversidade de abelhas sem ferrão em ambiente urbano no Brasil entre 1967 e 2019. Estados: BA=Bahia; GO= Goiás; $M A=$ Maranhão; $M G=$ Minas Gerais; $M T=$ Mato Grosso; $P A=$ Pará; $P E=$ Pernambuco; $P R=$ Paraná; $R S=$ Rio Grande do Sul; $S C=$ Santa Catarina; $S P=$ São Paulo. Métodos de Coleta: $A B=$ Armadilhas Atrativas; $A S=$ Busca Ativa; $M O=$ Möericke; $M T=$ Malaise; NS= Busca por Ninhos; TN= Ninhos-Armadilha. $*=$ gênero.

\begin{tabular}{|c|c|c|c|c|c|c|}
\hline Paper & Autor/Ano & Revista & Estado & $\begin{array}{c}\text { Duração } \\
\text { (Mês) }\end{array}$ & $\begin{array}{c}\text { Métodos } \\
\text { de } \\
\text { Coleta }\end{array}$ & $\begin{array}{c}\mathbf{N}^{\mathbf{o}} \text { de } \\
\text { Espécies }\end{array}$ \\
\hline 1 & Sakagami et al. 1967 [15] & J. Faculty of Science & PR & 12 & AS & 3 \\
\hline 32 & Sakagami e Laroca 1971 [47] & Kontyû & PR & 26 & AS & 6 \\
\hline 2 & Laroca et al. $1982[1]$ & Dusenia & PR & 12 & AS & 4 \\
\hline 3 & Taura e Laroca 1991 [21] & Acta Biológica Paranaense & PR & 6 & NS & 5 \\
\hline 186 & Knoll et al. 1993 [48] & Livro & SP & 14 & AS & 14 \\
\hline 31 & Bortoli e Laroca 1997 [33] & Acta Biológica Paranaense & PR & 13 & AS & 6 \\
\hline 24 & Carvalho e Marchini 1999 [49] & Rev. Agricultura & SP & 6 & NS & 10 \\
\hline 155 & Proní 2000 [50] & Arq Cienc Vet Zool & PR & 48 & NS & 19 \\
\hline 4 & Taura e Laroca 2001 [51] & Acta Biológica Paranaense & PR & 12 & AS & 5 \\
\hline 10 & Albuquerque et al. 2001 [52] & Acta Amazonica & MA & 12 & AS & 8 \\
\hline 130 & Sousa et al. 2002 [53] & Bioscience Journal & MG & 16 & NS & 4 \\
\hline 42 & Antonini e Martins 2003 [54] & Neotropical Entomology & MG & 12 & AS & 7 \\
\hline 66 & Toledo et al. 2003 [55] & Braz Arch Biol Technol & PR & 6 & AS & 2 \\
\hline 75 & Locatelli de Souza 2003 [56] & Tese & PE & 27 & AS & 5 \\
\hline 36 & Anacleto e Marchini 2005 [57] & Acta Sci. Biol Sci & SP & 12 & AS & 11 \\
\hline 88 & Souza at al. $2005[35]$ & Candombá & BA & 3 & NS & 5 \\
\hline 125 & Viana e Kleinert 2005 [58] & Biota Neotropica & BA & 12 & AS & 4 \\
\hline 168 & Zanette et al. 2005 [2] & Landscape Urban Planning & MG & 4 & AS & 8 \\
\hline 95 & Taura et. al. 2007 [5] & Acta Biológica Paranaense & PR & 12 & AS & 9 \\
\hline 115 & d'Avila e Marchini 2008 [59] & Ciência Florestal & SP & 12 & AS & 2 \\
\hline 180 & Alvarenga 2008 [60] & Dissertação & SP & - & $\mathrm{NS}, \mathrm{TN}$ & 3 \\
\hline 97 & Santiago et. al. 2009 [61] & Biota Neotropica & GO & 4 & AS & 21 \\
\hline 169 & Mateus et al. 2009 [62] & Mensagem Doce & MT & 12 & NS & 20 \\
\hline 11 & Gruchowski-W et al. 2010 [63] & Biodiversidade Pampeana & PR & 12 & $\mathrm{AB}, \mathrm{MT}$ & 3 \\
\hline 71 & Cure-Hakim e Laroca 2010 [64] & Acta Biológica Paranaense & PR & 13 & AS & 1 \\
\hline 128 & Steiner et al. 2010 [65] & Oecologia Australis & $\mathrm{SC}$ & 102 & AS & 11 \\
\hline 153 & Pioker-Hara 2011 [66] & Tese & SP & 29 & NS & 6 \\
\hline 56 & Antunes et al. 2012 [37] & Magistra & SP & 26 & AS & 7 \\
\hline 146 & Antonini et al. 2012 [38] & Urban Ecosystems & MG & 33 & AS & 23 \\
\hline 177 & Santos 2012 [67] & Dissertação & SP & 12 & AS & 7 \\
\hline 138 & Martins et al. 2013 [68] & Zoologia (Curitiba) & $\mathrm{PR}$ & 12 & AS & 3 \\
\hline 187 & Aidair et al 2013 [69] & Bioscience Journal & MG & 7 & NS & 7 \\
\hline 57 & Werneck e Faria-Mucci 2014 [70] & EntomoBrasilis & MG & 14 & NS & 9 \\
\hline 150 & Sousa 2014 [71] & Dissertação & SP & 27 & NS & 5 \\
\hline
\end{tabular}




\begin{tabular}{cllcccc} 
Table 1 (continuação) & Revista & Estado & $\begin{array}{c}\text { Duração } \\
\text { (Mês) }\end{array}$ & $\begin{array}{c}\text { Métodos } \\
\text { de } \\
\text { Coleta }\end{array}$ & $\begin{array}{c}\mathbf{N}^{\mathbf{o}} \text { de } \\
\text { Espécies }\end{array}$ \\
\hline 34 & Pereira 2015 [72] & Rev. Gestão Tecnol. Ciência & MG & 9 & AB & 2 \\
162 & Vieira 2015 [73] & Dissertação & MG & 3 & NS & 9 \\
98 & Sorreque 2016 [74] & Monografia & PR & 5 & AS & 6 \\
142 & Vieira et al. 2016 [22] & Sociobiology & MG & 2 & NS & 7 \\
101 & Possamai 2017 [75] & Acta Biológica Catarinense & SC & 28 & AS & 5 \\
148 & Mesquita et al. 2017 [19] & Agroecossistemas & PA & 11 & NS & 6 \\
\hline 35 & Somavilla et al. 2018 [39] & EntomoBrasilis & RS & 48 & AS & 12 \\
77 & Sobreiro 2018 [76] & Tese & PR & 12 & AS & 6 \\
116 & Silva 2019 [77] & Monografia & PR & 4 & MO & 2 \\
154 & Dantas e Silva et al. 2019 [78] & Magistra & BA & 12 & AS, NS, & $5^{*}$ \\
\hline & & & & & TN &
\end{tabular}

A primeira publicação envolvendo a presença de abelhas sem ferrão em ambiente urbano data de 1967 [15]. O Prof. Shôichi F. Sakagami natural de Chiba, Japão (1927-1996), Professor Emérito da Universidade de Hokkaido, tornou-se um entusiasta no estudo das abelhas indígenas do Brasil, atuando como Professor Visitante na Universidade Estadual Paulista (UNESP) em Rio Claro, SP (1961-63) e na Universidade de São Paulo em Ribeirão Preto, SP (1971-72) [28]. Sua estadia no Brasil foi muito produtiva, originando diversas publicações, além de estimular a nucleação de vários grupos de pesquisa com abelhas indígenas no Brasil e, sem dúvida, lançando um olhar pioneiro para a presença desses insetos em ambiente urbano. Sua contribuição para a ciência brasileira foi reconhecida em 1976, quando foi nomeado membro da Academia Brasileira de Ciências.

De 1971 a 1993, mais quatro estudos foram publicados pontualmente. A partir de 1997, as publicações começaram a ser mais frequentes, quase que anualmente, sendo 2003, 2005, 2010 e 2012 os anos mais produtivos. Na última década (2010-2019), 21 estudos foram realizados, representando 47,72\% do total de estudos realizados no Brasil (Figura 1). Segundo o Conselho Nacional de Desenvolvimento Científico e Tecnológico (CNPq), o número de grupos de pesquisa no Brasil vem crescendo constantemente desde o ano 2000, devido à expansão dos programas de pós-graduação no início do século, o que significa que há um número maior de pesquisadores executando estudos dentro do tema, o que provavelmente explica a maior produtividade durante os anos indicados.

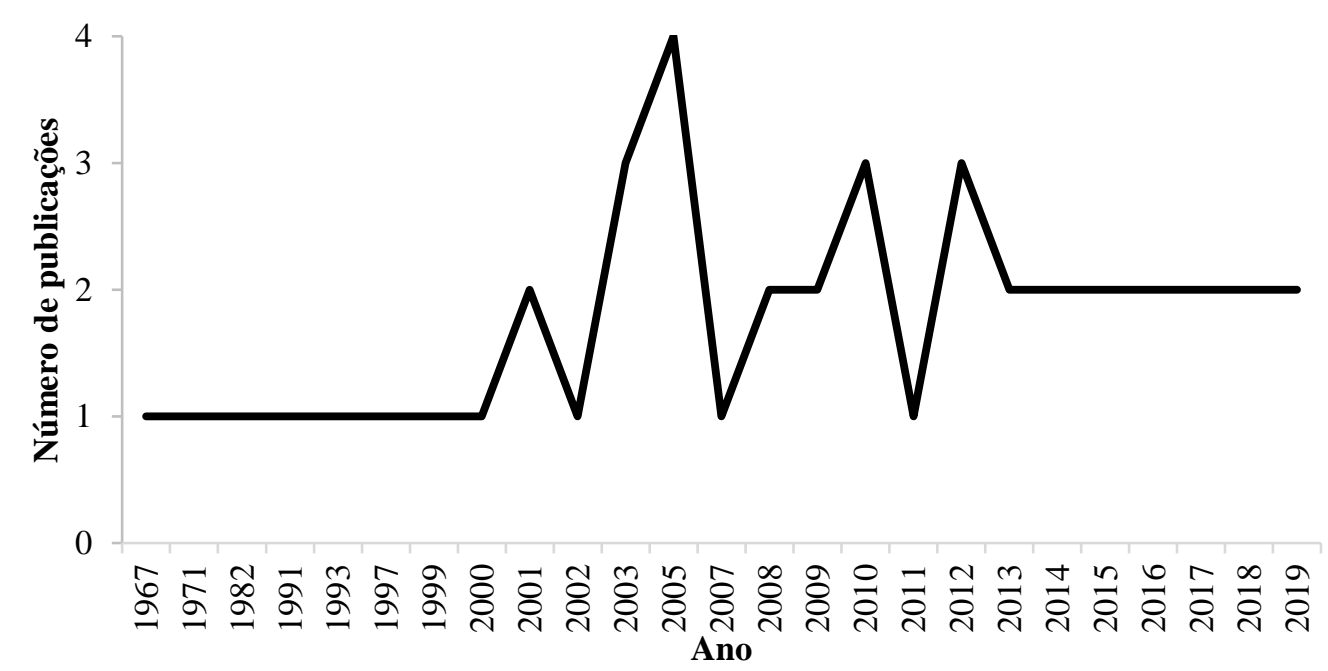

Figura 1: Número, por ano, de estudos de diversidade de abelhas sem ferrão em ambiente urbano entre 1967 e 2019. 
A distribuição dos estudos realizados no Brasil se concentrou nos estados do Paraná ( $\mathrm{n}=15$; $34,09 \%)$, Minas Gerais $(n=9 ; 20,45 \%)$ e São Paulo $(n=9 ; 20,45 \%)$, respectivamente, que juntos representam os locais onde foram realizados mais da metade de todos os estudos. Para o estado da Bahia, foram encontradas três publicações $(6,81 \%)$, seguida pelo estado de Santa Catarina, com duas publicações (4,54\%). Os estados de Goiás, Maranhão, Mato Grosso, Pará, Pernambuco, Rio Grande do Sul apresentaram apenas uma publicação cada, enquanto dezesseis estados ainda não possuem estudos de inventário de abelhas sem ferrão em ambiente urbano (Figura 2). A concentração dos trabalhos entre os estados do Paraná, Minas Gerais e São Paulo pode ser explicada por ser a região que abriga as escolas mais tradicionais e a maioria dos grupos de pesquisas sobre abelhas do Brasil, estabelecidos em universidades e institutos de pesquisas, dentre os quais devem ser destacados a Universidade Federal do Paraná, a Universidade de São Paulo e a Universidade Federal de Minas Gerais, que estão inseridas em grandes centros urbanos e, juntas, abrigam coleções de abelhas com relevância científica internacional.

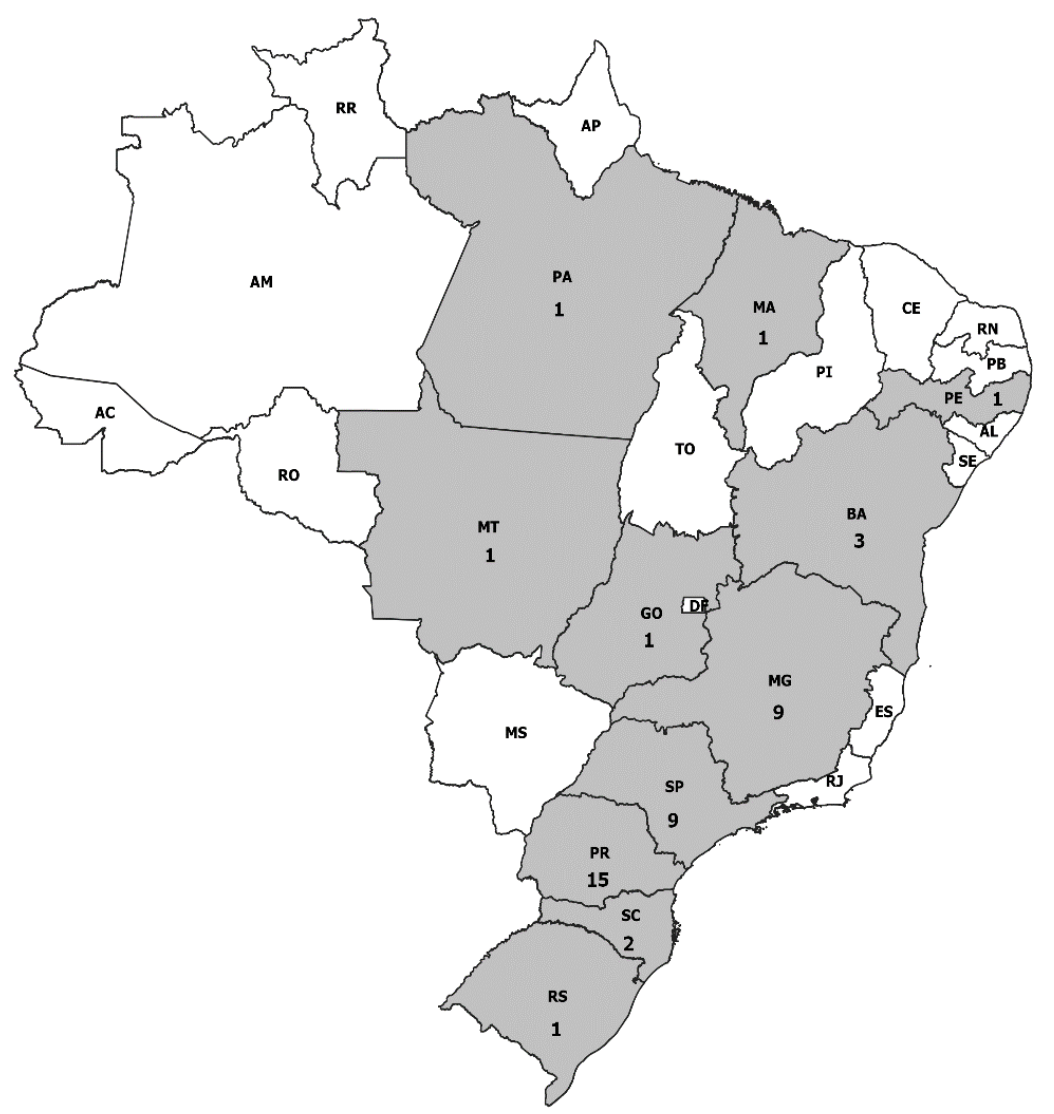

Figura 2: Representação geográfica do número de estudos de diversidade de abelhas sem ferrão em ambiente urbano entre 1967 e 2019.

Entretanto, apesar de localizados geograficamente próximos dos estados onde se há maior produção sobre a diversidade urbana de meliponíneos, os estados do Rio de Janeiro e Espirito Santo, surpreendentemente, não apresentaram estudos executados em seus territórios, que estão inseridos dentro do domínio Floresta Atlântica a nível do mar, indicando assim, prioridade de execução de futuros estudos a fim de se conhecer melhor a fauna urbana de abelhas sem ferrão associada a um dos biomas mais ameaçados e fragmentados do Brasil. A ausência de trabalhos na maioria dos estados das regiões Norte e Nordeste provavelmente se deve a priorização na realização de pesquisas em áreas naturais, visto a existência e interesse científico da Amazônia e Caatinga. No entanto, faz-se necessário investigar a urbanização e sua influência sobre as comunidades de meliponíneos presentes em localidades inseridas ou próximas a esses biomas. 
Quanto a duração da realização dos estudos, podemos observar que a maioria dos estudos foram realizados em períodos inferiores a 12 meses $(n=28)$ (Tabela 1), provavelmente por se tratarem de pesquisas incluídas em projetos de iniciação científica ou mestrado, cujo tempo de execução é limitado. Durações superiores a 12 meses foram encontradas em 15 trabalhos (de 13 a 102 meses). Apenas um trabalho não especifica o tempo de estudo empregado. Portanto, fica claro que não há padronização no tempo de duração dos estudos, o que seria importante para ajudar a fazer comparações mais precisas e detalhadas entre as publicações.

Das 44 publicações encontradas, apenas 16 focaram exclusivamente em inventariar a fauna de abelhas sem ferrão em suas respectivas áreas de estudo, enquanto os demais incluíram outros grupos de abelhas e vespas. No que diz respeito à amostragem, foram registrados seis métodos utilizados para coletar abelhas sem ferrão em ambiente urbano, sendo eles: Armadilhas Atrativas (Attractive Baits; $\mathrm{n}=2$ ), Busca Ativa (Active Search; $\mathrm{n}=27$ ), Malaise (Malaise Trap; $\mathrm{n}=1$ ), Möericke $(\mathrm{n}=1)$, Busca por Ninhos (Nest Search; $\mathrm{n}=15)$ e Ninhos-Armadilha (Trap Nests; $\mathrm{n}=2)$. A maioria dos estudos $(n=39 ; 88,63 \%)$ utilizou apenas um método de coleta, sendo "Busca Ativa" $(n=25)$ o mais utilizado, seguido de "Busca por Ninhos" $(n=13)$ e somente um estudo (Silva, 2019) que utilizou "Möericke" como método principal. Entretanto, o ideal seria que os trabalhos conciliassem e sistematizassem vários métodos a fim de obter melhor eficiência na amostragem, registrando assim, maior número de espécies de Meliponina em determinada área [29, 30, 31], assim como já foi registrado um acréscimo de espécies, devido ao consórcio de métodos, para outros Hymenoptera, como as vespas sociais [32]. Contudo, vale destacar que a elaboração e utilização de métodos devem ser baseadas na biologia e ecologia dos animais estudados a fim de evitar um esforço ineficaz durante a realização do estudo.

Vale destacar também a importância do método "Busca por Ninhos", que consiste na inspeção de áreas em busca de colônias de meliponíneos, pois através de sua execução é possível adquirir informações importantes sobre a ecologia de nidificação do grupo em ambiente urbano. Assim, os estudos nos forneceram informações sobre o substrato utilizado para nidificação, sobre a altura da entrada do ninho em relação ao solo e acerca da posição da entrada, em relação aos pontos cardeais, das colônias amostradas (Tabela 2). Foi possível identificar três tipos de substratos utilizados pelas espécies entre os estudos, sendo eles: Natural (Árvores e Solo), Artificial (Construções Humanas) e Termiteiro. Acerca da altura onde os ninhos estavam localizados, foram registrados desde ninhos subterrâneos, alguns localizados a poucos centímetros acima do solo e até ninhos localizados em copas de árvores (Tabela 2).

A respeito da utilização de índices ecológicos entre os estudos, verificamos que Bortoli e Laroca (1997) [33] foi o primeiro estudo a utilizar tais índices, no caso o índice de Sorensen, para verificar a similaridade entre a melissofauna de áreas urbanas com sua área de estudo em Guarapuava dentro do estado do Paraná. Ao todo, 26 (59,09\%) estudos utilizaram ao menos um índice ecológico em suas análises, sendo o índice de Shannon (H') o mais utilizado entre os estudos $(n=19)$.

Foram registradas 60 espécies, pertencentes a 21 gêneros, dentro os quais foram mais representativos os gêneros Trigona (onze espécies), Melipona (sete espécies) e Plebeia (sete espécies). A constância calculada mostrou que das 60 espécies registradas entre as publicações, 53 $(88,33 \%)$ são consideradas acidentais, cinco foram consideradas acessórias $(8,33 \%)$ e apenas duas (3,33\%) foram consideradas constantes sendo elas Trigona spinipes (Fabricius, 1793) e Tetragonisca angustula (Latreille, 1811), presentes em 88,64\% e 79,55\% dos trabalhos, respectivamente. A constância de T. spinipes e T. angustula entre os estudos provavelmente significa que elas são as espécies de Meliponina mais generalistas [34, 35, 36, 37, 38, 39] e amplamente distribuídas pelo território brasileiro [40], seja devido à sua ecologia de nidificação e dispersão, comportamentos de forrageio e defesa ou relação com a exploração humana de seus recursos. Através da utilização de chaves taxonômicas, comparação com exemplares presentes em coleções científicas ou envio de espécimes a especialistas em taxonomia de abelhas, todos os trabalhos identificaram a maioria das espécies de abelhas sem ferrão coletadas, com exceção de apenas um estudo.

Em relação a distribuição das espécies registradas por estado, verificamos que seis $(10,16 \%)$ espécies estavam distribuídas em mais de cinco estados amostrados, sendo elas: T. spinipes $(\mathrm{n}=10)$, T. angustula $(\mathrm{n}=9)$, Plebeia droryana (Friese, 1900) $(\mathrm{n}=6)$, Tetragona clavipes (Fabricius, 1804) $(\mathrm{n}=6)$, Scaptotrigona bipunctata (Lepeletier, 1836) $(\mathrm{n}=5)$, Scaptotrigona postica (Latreille, 1807) 
$(\mathrm{n}=5)$ e Schwarziana quadripunctata (Lepeletier, 1836) $(\mathrm{n}=5)$. Contudo, $28(46,6 \%)$ espécies foram registradas pontualmente em um único estado. Apesar disso, o estado com o maior número de espécies registradas é São Paulo ( $n=24)$, enquanto que Pernambuco $(n=4)$ foi o estado com o menor número de espécies registradas, isso porque apenas um único estudo foi realizado no estado. Sabese que a riqueza de espécies de Apidae tende a aumentar em direção a Linha do Equador [41], portanto acredita-se que os estados das regiões Norte e Nordeste do Brasil também apresentem um número significativo de espécies em ambiente urbano, contudo o número reduzido de estudos nesses locais dificulta a compreensão de quantas e quais espécies estão estabelecidas nesse tipo de ambiente. Tendo como exemplo o estado do Amazonas que, apesar de abrigar a maior diversidade de abelhas sem ferrão já inventariada no Brasil [29, 42], não foi representado nessa análise devido à ausência de estudos em centros urbanos. 
Tabela 2: Publicações, meio de publicação, duração de estudo, métodos de coleta, área de estudo (estado) e número de espécies registrados em estudos de diversidade de abelhas sem ferrão em ambiente urbano no Brasil entre 1967 e 2019. Estados: BA=Bahia; GO= Goiás; MA= Maranhão; MG= Minas Gerais; MT= Mato Grosso; PA= Pará; PE= Pernambuco; PR= Paraná; $R S=$ Rio Grande do Sul; $S C=$ Santa Catarina; SP= São Paulo. Métodos de Coleta: AB= Armadilhas Atrativas; AS= Busca Ativa; $M O=$ Möericke $; M T=$ Malaise $;$ NS= Busca por Ninhos; $T N=$ Ninhos-Armadilha.$*$ gênero.

\begin{tabular}{|c|c|c|c|c|c|c|}
\hline Espécie & Referências & $\begin{array}{l}\text { Estado do } \\
\text { Estudo }\end{array}$ & $\begin{array}{l}\text { Métodos de } \\
\text { Coleta }\end{array}$ & $\begin{array}{c}\text { Substrato } \\
\text { de } \\
\text { Nidificação }\end{array}$ & $\begin{array}{l}\text { Amplitude } \\
\text { Altura (m) } \\
\text { de Ninhos } \\
\end{array}$ & Constância \\
\hline Celetrigona longicornis (Friese, 1903) & 169 & MT & NS & $\mathrm{A}, \mathrm{N}$ & - & 2,27 \\
\hline Cephalotrigona capitata (Smith, 1854) & 36,146 & MG, SP & AS & - & - & 4,55 \\
\hline Friesella schrottkyi (Friese, 1900) & $24,57,162,168,186$ & MG, SP & AS, NS & $\mathrm{A}, \mathrm{N}$ & $0,45-1,28$ & 11,36 \\
\hline Frieseomelitta doederleini (Friese, 1900) & 125 & BA & AS & - & - & 2,27 \\
\hline Frieseomelitta languida Moure, 1990 & 125 & BA & AS & - & - & 2,27 \\
\hline Frieseomelitta silvestrii (Friese, 1902) & $97,146,169$ & $\mathrm{GO}, \mathrm{MG}, \mathrm{MT}$ & AS, NS & $\mathrm{A}, \mathrm{N}$ & - & 6,82 \\
\hline Frieseomelitta varia (Lepeletier, 1836) & 97,169 & GO, MT & AS, NS & A, N & - & 4,55 \\
\hline Geotrigona mombuca (Smith, 1863) & 98,146 & MG, PR & AS & - & - & 4,55 \\
\hline Geotrigona subterranea (Friese, 1901) & $42,146,168$ & MG & AS & - & - & 6,82 \\
\hline Lestrimelitta limao (Smith, 1863) & $3,4,169$ & MT, PR & NS & $\mathrm{N}$ & - & 6,82 \\
\hline Leurotrigona muelleri (Friese, 1900) & $36,95,153,177$ & PR, SP & AS, NS & $\mathrm{N}$ & $0,75-1,80$ & 9,09 \\
\hline Melipona bicolor Lepeletier, 1836 & 57,146 & MG & AS, NS & $\mathrm{N}$ & - & 4,55 \\
\hline Melipona fasciculata Smith, 1854 & 10 & MA & AS & - & - & 2,27 \\
\hline Melipona interrupta Latreille, 1811 & 148 & PA & NS & $\mathrm{N}$ & $0,95-6$ & 2,27 \\
\hline Melipona marginata Lepeletier, 1836 & $35,128,186$ & RS, SC, SP & AS & - & - & 6,82 \\
\hline Melipona quadrifasciata Lepeletier, 1836 & $\begin{array}{c}31,32,36,42,128,138,142,146,153 \\
162,186\end{array}$ & PR, MG, SC, SP & AS, NS & $\mathrm{N}$ & $1,67-3$ & 25,00 \\
\hline Melipona scutellaris Latreille, 1811 & 75 & PE & AS & - & - & 2,27 \\
\hline Melipona seminigra Friese, 1903 & 148 & PA & NS & $\mathrm{N}$ & 2,33 & 2,27 \\
\hline Mourella caerulea (Friese, 1900) & 35 & RS & AS & - & - & 2,27 \\
\hline Nannotrigona testaceicornis (Lepeletier, 1836) & $\begin{array}{c}24,36,42,56,88,95,98,130,142,146 \\
155,162,168,186,187\end{array}$ & $\mathrm{BA}, \mathrm{MG}, \mathrm{PR}, \mathrm{SP}$ & AS, NS & $\mathrm{A}, \mathrm{N}$ & $0,37-2,96$ & 34,09 \\
\hline Oxytrigona tataira (Smith, 1863) & $11,88,169$ & BA, PR, MT & $\mathrm{AB}, \mathrm{MT}, \mathrm{NS}$ & $\mathrm{N}$ & $0,30-5$ & 6,82 \\
\hline
\end{tabular}


Table 2 (continuação)

\begin{tabular}{|c|c|c|c|c|c|c|}
\hline Espécie & Referências & $\begin{array}{l}\text { Estado do } \\
\text { Estudo }\end{array}$ & $\begin{array}{l}\text { Métodos de } \\
\text { Coleta }\end{array}$ & $\begin{array}{c}\text { Substrato } \\
\text { de } \\
\text { Nidificação }\end{array}$ & $\begin{array}{l}\text { Amplitude } \\
\text { Altura (m) } \\
\text { de Ninhos }\end{array}$ & Constância \\
\hline Paratrigona lineata (Lepeletier, 1836) & $36,42,97,146,168,177$ & GO, MG, SP & AS & - & - & 13,64 \\
\hline Paratrigona subnuda Moure, 1947 & $146,150,186$ & $\mathrm{MG}, \mathrm{SP}$ & AS, NS & $\mathrm{N}$ & - & 6,82 \\
\hline Partamona ailyae Camargo, 1980 & 169 & MT & NS & $\mathrm{T}$ & - & 2,27 \\
\hline Partamona criptica Pedro \& Camargo, 2003 & 128 & $\mathrm{SC}$ & AS & - & - & 2,27 \\
\hline Partamona cupira (Smith, 1863) & 186 & SP & AS & - & - & 2,27 \\
\hline Partamona helleri (Friese, 1900) & $24,42,101,128,142,146,162,168$ & $\mathrm{MG}, \mathrm{SC}, \mathrm{SP}$ & AS, NS & $\mathrm{A}, \mathrm{N}$ & $1,10-6,44$ & 18,18 \\
\hline Partamona vicina Camargo, 1980 & 169 & MT & NS & $\mathrm{T}$ & - & 2,27 \\
\hline Plebeia droryana (Friese, 1900) & $\begin{array}{c}24,35,36,56,77,88,95,128,146,153 \\
155,168,177,186,187\end{array}$ & $\begin{array}{l}\text { BA, MG, PR, RS, } \\
\text { SC, SP }\end{array}$ & AS, NS & $\mathrm{A}, \mathrm{N}$ & $0,50-3,80$ & 34,09 \\
\hline Plebeia emerina (Friese, 1900) & $2,3,4,31,32,35,71,101,128,186$ & PR, RS, SC, SP & AS, NS & $\mathrm{A}, \mathrm{N}$ & $0,31-3,83$ & 22,73 \\
\hline Plebeia minima (Gribodo, 1893) & 75 & $\mathrm{PE}$ & AS & - & - & 2,27 \\
\hline Plebeia nigriceps (Friese, 1901) & 35 & $\mathrm{RS}$ & AS & - & - & 2,27 \\
\hline Plebeia remota (Holmberg, 1903) & $35,98,128,186$ & $\mathrm{RS}, \mathrm{SC}$ & AS & - & - & 9,09 \\
\hline Plebeia saiqui (Friese, 1900) & 186 & SP & AS & - & - & 2,27 \\
\hline Plebeia wittmanni Moure \& Camargo, 1989 & 35 & RS & AS & - & - & 2,27 \\
\hline Scaptotrigona bipunctata (Lepeletier, 1836) & $2,3,4,31,32,35,95,98,128,150,155$ & $\begin{array}{l}\text { MG, PR, RS, SC, } \\
\text { SP }\end{array}$ & AS, NS & $\mathrm{N}$ & $0,38-1,50$ & 27,27 \\
\hline Scaptotrigona depilis (Moure, 1942) & $56,97,187$ & GO, MG, SP & AS, NS & $\mathrm{N}$ & $1,16-2,44$ & 6,82 \\
\hline Scaptotrigona polysticta Moure, 1950 & 24 & SP & NS & - & $1,65-1,75$ & 2,27 \\
\hline Scaptotrigona postica (Latreille, 1807) & $97,146,155,169,186$ & $\begin{array}{l}\mathrm{GO}, \mathrm{MG}, \mathrm{MT} \\
\quad \mathrm{PR}, \mathrm{SP}\end{array}$ & AS, NS & $\mathrm{N}$ & - & 11,36 \\
\hline Scaptotrigona xanthotricha Moure, 1950 & 162 & MG & NS & $\mathrm{N}$ & $0.58-3,32$ & 2,27 \\
\hline Scaura latitarsis (Friese, 1900) & 97,180 & $\mathrm{GO}, \mathrm{SP}$ & AS, NS & $\mathrm{T}$ & - & 4,55 \\
\hline Scaura longula (Lepeletier, 1836) & 97,169 & GO, MT & AS, NS & $\mathrm{N}$ & - & 4,55 \\
\hline Schwarziana quadripunctata (Lepeletier, 1836) & $11,32,35,36,77,128,162,186$ & $\begin{array}{l}\text { MG, PR, RS, SC, } \\
\text { SP }\end{array}$ & AS, MT, NS & $\mathrm{N}$ & 1,75 & 18,18 \\
\hline
\end{tabular}


Table 2 (continuação)

\begin{tabular}{|c|c|c|c|c|c|c|}
\hline Espécie & Referências & $\begin{array}{l}\text { Estado do } \\
\text { Estudo }\end{array}$ & $\begin{array}{c}\text { Métodos de } \\
\text { Coleta }\end{array}$ & $\begin{array}{c}\begin{array}{c}\text { Substrato } \\
\text { de }\end{array} \\
\text { Nidificação } \\
\end{array}$ & $\begin{array}{l}\text { Amplitude } \\
\text { Altura (m) } \\
\text { de Ninhos } \\
\end{array}$ & Constância \\
\hline Tetragona clavipes (Fabricius, 1804) & $\begin{array}{c}56,57,77,95,97,142,146,148,155 \\
162,169,187\end{array}$ & $\begin{array}{l}\text { GO, MG, MT, } \\
\text { PA, PR, SP }\end{array}$ & AS, NS & $\mathrm{N}$ & $0,57-6,00$ & 27,27 \\
\hline Tetragona dorsalis (Smith, 1854) & 10 & MA & AS & - & - & 2,27 \\
\hline Tetragona quadrangula (Lepeletier, 1836) & 57 & MG & NS & $\mathrm{N}$ & - & 2,27 \\
\hline Tetragonisca angustula (Latreille, 1811) & $\begin{array}{c}2,3,4,24,31,34,35,36,42,56,57,66 \\
75,77,88,95,97,98,101,128,130,138 \\
142,146,150,153,154,155,162,168 \\
169,177,180,186,187\end{array}$ & $\begin{array}{l}\text { BA, GO, MG, } \\
\text { MT, PE, PR, RS, } \\
\text { SC, SP }\end{array}$ & $\begin{array}{c}\mathrm{AB}, \mathrm{AS}, \mathrm{NS}, \\
\mathrm{TN}\end{array}$ & $\mathrm{A}, \mathrm{N}, \mathrm{T}$ & $0,09-7$ & 79,55 \\
\hline Tetragonisca fiebrigi (Schwarz, 1938) & 116 & PR & MO & - & - & 2,27 \\
\hline Trigona branneri Cockerell, 1912 & 95 & PR & AS & - & - & 2,27 \\
\hline Trigona braueri Friese, 1900 & 101,150 & $\mathrm{SC}, \mathrm{SP}$ & AS, NS & $\mathrm{N}$ & - & 4,55 \\
\hline Trigona chanchamayoensis Schwarz, 1948 & 97 & GO & AS & - & - & 2,27 \\
\hline Trigona fulviventris Guérin, 1844 & $10,97,146$ & $\mathrm{GO}, \mathrm{MA}, \mathrm{MG}$ & AS & - & - & 6,82 \\
\hline Trigona fuscipennis Friese, 1900 & $10,95,97,169$ & $\mathrm{GO}, \mathrm{MA}, \mathrm{MT}, \mathrm{PR}$ & AS, NS & $\mathrm{N}$ & - & 9,09 \\
\hline Trigona guianae Cockerell, 1910 & 148 & PA & NS & $\mathrm{N}$ & - & 2,27 \\
\hline Trigona hyalinata (Lepeletier, 1836) & $24,36,56,57,97,146$ 153, 177, 180, 187 & $\mathrm{GO}, \mathrm{MG}, \mathrm{SP}$ & AS, NS & $\mathrm{A}, \mathrm{N}$ & $4,50-7,00$ & 22,73 \\
\hline Trigona pallens (Fabricius, 1798) & $10,148,169$ & MA, MT, PA & AS, NS & $\mathrm{A}, \mathrm{N}, \mathrm{T}$ & $0,20-1,02$ & 6,82 \\
\hline Trigona recursa Smith, 1863 & 97,169 & GO, MT & AS, NS & $\mathrm{N}, \mathrm{T}$ & - & 4,55 \\
\hline Trigona spinipes (Fabricius, 1793) & $\begin{array}{c}1,2,3,4,10,11,24,31,32,34,35,36 \\
42,56,57,66,75,77,88,95,97,98,101 \\
115,116,125,128,130,138,142,146 \\
150,153,155,168,169,177,186,187\end{array}$ & $\begin{array}{l}\text { BA, GO, MA, } \\
\text { MG, MT, PE, PR, } \\
\text { RS, SC, SP }\end{array}$ & $\begin{array}{c}\mathrm{AB}, \mathrm{AS}, \mathrm{MT} \\
\mathrm{MO}, \mathrm{NS}\end{array}$ & $\mathrm{N}$ & $0,75-18,00$ & 88,64 \\
\hline Trigona truculenta Almeida, 1984 & 97 & GO & AS & - & - & 2,27 \\
\hline Trigonisca nataliae (Moure, 1950) & 97,169 & GO, MT & AS & - & - & 4,55 \\
\hline
\end{tabular}


Baseado na curva de acumulação, foram registradas 83 espécies para Jackknife $1^{\circ}$ e 97 espécies para Jackknife $2^{\circ}$ (Figura 3), entretanto, os estudos foram capazes de registrar somente $72 \%$ e $62 \%$ das espécies estimadas para matrizes urbanas do Brasil. Esse resultado provavelmente se deve ao fato de que a maioria dos estudos foram concentrados em poucos pontos das regiões Sul e Sudeste do Brasil, como já comentado anteriormente, o que subestima a contribuição de algumas microrregiões, outras regiões e estados. Como é o caso do estado de Minas Gerais que, apesar de ter uma boa representação de estudos, estes estão em maioria localizados em municípios dentro do domínio Floresta Atlântica, afetando assim a representatividade da diversidade de espécies de outros domínios dentro do estado, como Caatinga [43] e Cerrado [44, 45, 46].

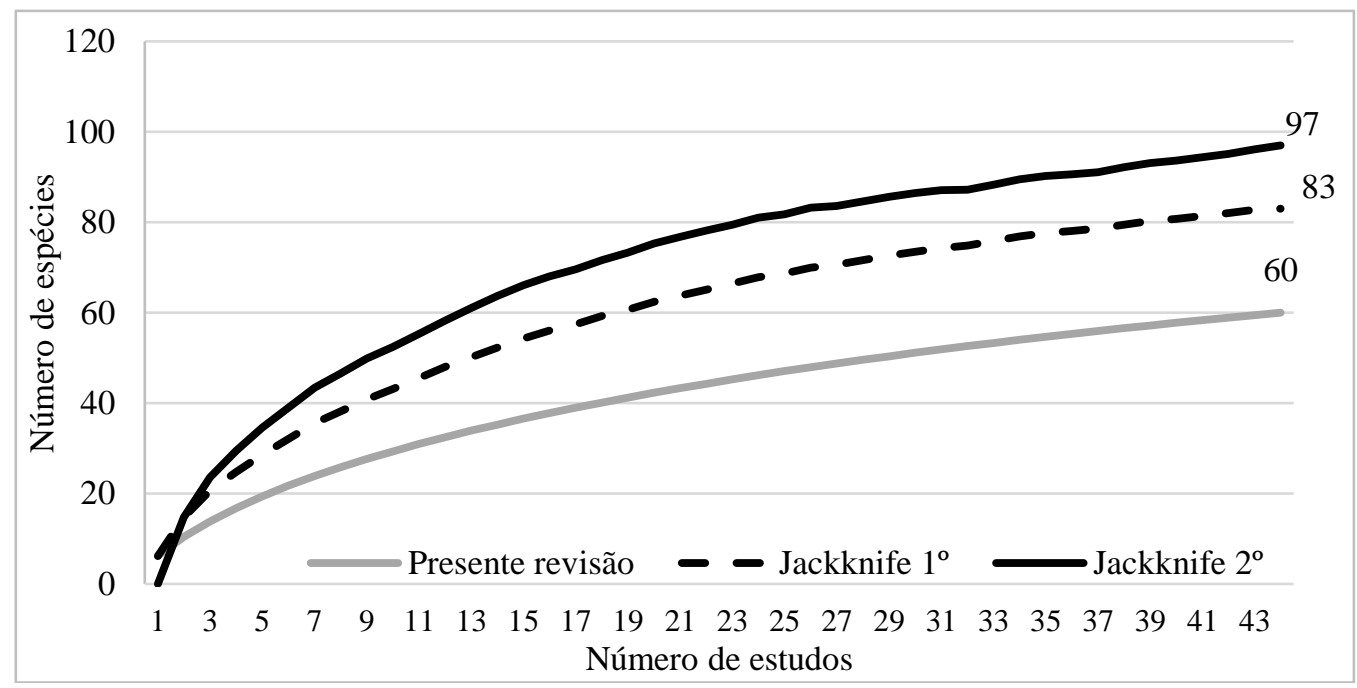

Figura 3: Curvas de Rarefação para estimadores de riqueza de espécies e Curvas de Acumulação de Espécies geradas por meio de estudos de diversidade de abelhas sem ferrão no Brasil de 1967 a 2019.

\section{CONCLUSÃO}

Reconhecido o papel ecológico de Meliponina e a adaptação de algumas espécies ao ambiente urbano, nós podemos dizer que ainda são muito poucos os estudos visando estudar a presença e ecologia do grupo em ambientes urbanos. A maioria dos estudos existentes são limitados a exploração de recursos pelas abelhas e seus hábitos de nidificação, conforme demostrado por nossa análise. Segundo Hernandez, Frankie e Thorp (2009) [3], seria interessante que futuros estudos abordassem análises de dinâmica da comunidade de abelhas urbanas para determinar relações causais entre a significância ecológica das características dos hábitats urbanos e sua utilização pelas abelhas. Vale salientar a importância da execução desses estudos para obter informações necessárias para a conservação e manejo das espécies de abelhas sem ferrão em um ambiente tão desafiador quanto o urbano. Portanto, nosso estudo buscou contribuir com o entendimento sobre a fauna de abelhas urbanas, com a indicação de potenciais locais para a execução de futuros estudos dentro o tema, demonstração dos métodos de coleta mais utilizados e espécies mais encontradas nesses ambientes, através de todo o material listado e presente em nossa análise.

\section{AGRADECIMENTOS}

Os autores gostariam de agradecer à Laura Oliveira Pires e à Anthoely Vieira Evangelista pelo auxílio durante a extração e análise dos dados. Também gostaríamos de agradecer aos professores Dr. Fernando A. Silveira, Dr. Celso Feitosa Martins, Dra. Silvia Regina de Menezes Pedro, Dra. Georgina Faria Mucci, Dr. Sidnei Mateus e Dra. Ana Maria Coelho Carvalho por enviarem manuscritos essenciais para a execução do estudo. O trabalho contou com o apoio da Universidade Federal de Juiz de Fora (UFJF), Coordenação de Aperfeiçoamento de Pessoal de Nível Superior 
(CAPES), Conselho de Desenvolvimento Científico e Tecnológico (CNPq) e Fundação de Amparo à Pesquisa do Estado de Minas (FAPEMIG).

\section{REFERÊNCIAS BIBLIOGRÁFICAS}

1. Laroca S, Cure JR, Bortoli CD. A associação de abelhas silvestres (Hymenoptera, Apoidea) de uma área restrita no interior da cidade de Curitiba (Brasil): uma abordagem biocenótica. Dusenia. 1982;13(3):93117.

2. Zanette LRS, Martins RP, Ribeiro SP. Effects of urbanization on Neotropical wasp and bee assemblages in a Brazilian metropolis. Landscape Urban Plann. 2005;71(2-4):105-121, doi: 10.1016/j.landurbplan.2004.02.003.

3. Hernandez JL, Frankie GW, Thorp RW. Ecology of urban bees: a review of current knowledge and directions for future study. Cities Environm (CATE). 2009;2(1):15, Article 3, doi: 10.15365/CATE.2132009.

4. Sánchez-Bayo F, Wyckhuys KA. Worldwide decline of the entomofauna: A review of its drivers. Biol Conserv. 2019;232:8-27, doi: 10.1016/j.biocon.2019.01.020.

5. Taura HM, Laroca S, Barbosa JF, Rodrigues J. Melissocenótica (Hymenoptera, Anthophila) no Parque Florestal dos Pioneiros, Maringá, PR. (sul do Brasil) - I. Abundância relativa e diversidade. Acta Biol. Par. 2007;36(1-2):47-65.

6. Maciel TT, Barbosa BC, Prezoto F. Urbanização e os Insetos Sociais. In: Andriolo A, Prezoto F, Barbosa BC, editores. Impactos Antrópicos: Biodiversidade Aquática \& Terrestre. Juiz de Fora: Real Consultoria em Negócios Ltda; 2018. p. 6-17.

7. Cockerell TDA. Descriptions of new bees collected by H. H. Smith in Brazil. Proceed Acad Nat Sci Philadelphia. 1900;52:356-377.

8. Ducke A. Beobachtungen Über Blutenbesuch, Erscheinungszeit etc. der bei Pará Vorkommenden - II Allgemeine Zeitschrift für Entomologie. 1902;17:321-326, 19: 400-405, 20:417-422.

9. Ducke A. Neue Beobachtungen über die Bienen der Amasonslander. Allgemeine Zeitschrift für Entomologie. 1906;2:51-60.

10. Ducke A. Contribution a la conuissance des Hymenopteres des deux Amériques. Rev d'Entomologie. 1908;27:28-87.

11. Ducke A. Contribution à la connaissance de la faune hymenénoptèrologique du Nordest du Brésil - I. Rev d'Entomologie. 1907;26:73-96.

12. Ducke A. Explorações botânicas e entomológicas no Estado do Ceará. Rev Trimestral Inst Ceará. 1910;24:3-61.

13. Friese H. Neue Bienenarten aus Süd-Amerika. Deutsche Entomol Zeitschrift. 1910;56:693-711.

14. Schrottky C. Ensaio sobre as abelhas do Brasil. Rev Museu Paulista. 1902;5:330-613.

15. Sakagami SF, Laroca S, Moure JS. Wild bee biocenotics in São José dos Pinhais (PR) south Brazil. Preliminary report. J Fac Sci Hokkaido Univ., Ser. Zool. 1967;16:253-291.

16. Silveira FA, Melo GAR, Almeida EAB. Abelhas brasileiras: sistemática e identificação. Belo Horizonte: Fernando A. Silveira; 2002. 253 p.

17. Kerr WE, Carvalho GA, Nascimento VA. Abelha uruçu: biologia, manejo e conservação. Vol. 2. Belo Horizonte: Fundação Acangaú; 1996. 144 p.

18. Martins CF, Laurino MC, Koedam D, Imperatriz-Fonseca VL. Espécies arbóreas utilizadas para nidificação por abelhas sem ferrão na caatinga (Seridó, PB; João Câmara, RN). Biota Neotr. 2004;4(2):18, doi: 10.1590/S1676-06032004000200003.

19. Mesquita NS, dos Santos GC, Mesquita NS, Mesquita RS, Mesquita FS, Rode R, Ribeiro RS, da Silva ASL. Diagnóstico da relação entre a arborização e a diversidade de abelhas sem ferrão (Apidae: Meliponini) no campus Tapajós e no Bosque Mekdece localizados em Santarém, PA. Rev Agroecossistemas. 2017;9(2):130-147, doi: 10.18542/ragros.v9i2.5096

20. Kerr WE, Sakagami SF, Zucchi R, Portugal Araujo V, Camargo JMF. Observações sobre a arquitetura dos ninhos e comportamento de algumas espécies de abelhas sem ferrão das vizinhanças de Manaus, Amazonas (Hymenoptera, Apoidea). Atas do Simpósio sobre a Biota Amazônica. 1967;5:235-309.

21. Taura HM, Laroca S. Abelhas altamente sociais (Apidae) de uma área restrita em Curitiba (Brasil): Distribuição dos ninhos e abundância relativa. Acta Biol Paranaense. 1991;20(1-4):85-101.

22. Vieira KM, Netto P, Amaral DL, Mendes SS, Castro LC, Prezoto F. Nesting stingless bees in urban areas: a reevaluation after eight years. Sociobiology. 2016;63(3):976-981, doi: 10.13102/sociobiology.v63i3.778. 
23. Moher D, Liberati A, Tetzlaff J, Altman DG, Prisma Group. Preferred reporting items for systematic reviews and meta-analyses: the PRISMA statement. PLoS med. 2009;6(7):e1000097, doi: 10.1371/journal.pmed.1000097.

24. Moher D, Shamseer L, Clarke M, Ghersi D, Liberati A, Petticrew M, Shekelle P, Stewart LA, Prisma-P Group. Preferred reporting items for systematic review and meta-analysis protocols (PRISMA-P) 2015 statement. Syst Rev. 2015;4(1):1, doi: 10.1186/2046-4053-4-1.

25. Bodenheimer FS. Precis d'écologie animale. Paris: Payot; 1955. 315 p.

26. Gotelli NJ, Colwell RK. Quantifying biodiversity: procedures and pitfalls in the measurement and comparison of species richness. Ecol Lett. 2001;4:379-391, doi: 10.1046/j.1461-0248.2001.00230.x.

27. Colwell RK, Elsensohn JE. EstimateS turns 20: statistical estimation of species richness and shared species from samples, with non-parametric extrapolation. Ecography. 2014;37(6):609-613, doi: 10.1111/ecog.00814.

28. Chino H, Toda MJ."OBITUARY," Zoological Sci. 1997;14(2):185-186, doi: 10.2108/zsj.14.185.

29. Oliveira ML, Morato EF, Garcia MVB. Diversidade de espécies e densidade de ninhos de abelhas sociais sem ferrão (Hymenoptera, Apidae, Meliponinae) em floresta de terra firme na Amazônia central. Rev Bras Zool. 1995;12(1):13-4, doi: 10.1590/S0101-81751995000100004.

30. Pinheiro-Machado C, Silveira FA. Surveying and monitoring of pollinators in natural landscapes and in cultivated fields. In: Imperatriz-Fonseca VL, Saraiva AM, De Jong D, editores. Bees as pollinators in Brazil: Assessing the status and suggesting best practices. Ribeirão Preto: Holos; 2006. p. 25-37.

31. Almeida RPS, Arruda FV, Silva DP, Coelho BWT. Bees (Hymenoptera, Apoidea) in an Ecotonal Cerrado-Amazon Region in Brazil. Sociobiology. 2019;66(3):457-466, doi: 10.13102/sociobiology.v66i3.3463.

32. Barbosa BC, Detoni M, Maciel TT, Prezoto F. Studies of social wasp diversity in Brazil: Over 30 years of research, advancements and priorities. Sociobiology. 2016;63(3):858-880, doi: 10.13102/sociobiology.v63i3.1031

33. Bortoli C, Laroca S. Melissocenologia no Terceiro Planalto Paranaense. I: Abundância relativa das abelhas silvestres (Apoidea) de um biótopo urbano de Guarapuava (PR, Brasil). Acta Biol Paranaense. 1997;26(1-4):51-86.

34. Silveira FA, Campos MJO. A melissofauna de Corumbataí (SP) e Paraopeba (MG) e uma análise da biogeografia das abelhas do cerrado brasileiro (Hymenoptera, Apoidea). Rev Bras Entomol. 1995;39(2):371-401.

35. Souza SGX, Melo AMC, das Neves EL, Teixeira A. As abelhas sem ferrão (Apidae: Meliponina) residentes no campus Federação/Ondina da Universidade Federal da Bahia, Salvador, Bahia, Brasil. Cadombá - Rev Virtual. 2005;1(1):57-69.

36. Araújo VA, Antonini Y, Araújo A. Diversity of bees and their floral resources at altitudinal areas in the Southern Espinhaço Range, Minas Gerais, Brazil. Neotr Entomol. 2006;35(1):30-40, doi: 10.1590/S1519$566 \times 2006000100005$.

37. Antunes HA, Nunes LA, Silva JD, Marchini LC. Abelhas nativas (Apidae: Meliponina) e seus recursos florais em um fragmento de mata localizado em área urbana. Magistra. 2012;24(1):7-14.

38. Antonini Y, Martins RP, Aguiar LM, Loyola RD. Richness, composition and trophic niche of stingless bee assemblages in urban forest remnants. Urban Ecosyst. 2012;16(3):527-541, doi: 10.1007/s11252012-0281-0.

39. Somavilla A, Schoeninger K, Nogueira DS, Kohler A. Diversidade de abelhas (Hymenoptera: Apoidea) e visitação floral em uma área de Mata Atlântica no Sul do Brasil. EntomoBrasilis. 2018;11(3):191-200, doi: 10.12741/ebrasilis.v11i3.800.

40. Moure JS, Urban D, Melo GAR, organizers. Catalogue of Bees (Hymenoptera, Apoidea) in the Neotropical Region. Curitiba: Sociedade Brasileira de Entomologia; 2007. 1058 p.

41. Roubik DW. Ecology and natural history of tropical bees. Cambridge: Cambridge University Press; 1989. $514 \mathrm{p}$.

42. Oliveira FFD, Richers BTT, Silva JRD, Farias RC, Matos, TADL. Guia ilustrado das abelhas" sem ferrão" das Reservas Amanã e Mamirauá, Amazonas, Brasil (Hymenoptera, Apidae, Meliponini). Tefé: Instituto de Desenvolvimento Sustentável Mamirauá; 2013. 267 p.

43. Alvarenga AS. Influência da estrutura da vegetação na comunidade de abelhas em florestas tropicais secas [dissertação]. Minas Gerais (BR): Universidade Federal de Minas Gerais; 2014. 47 p.

44. Carvalho AMC, Bego LR. Studies of Apoidea fauna of cerrado vegetation at the Panga Ecological Reserve, Uberlândia, MG, Brazil. Rev Bras Entomol. 1996;40(1):147-156.

45. Azevedo AA. Composição de faunas de abelhas (Hymenoptera, Apoidea) e da flora associada em área de cerrado de Minas Gerais, Brasil [tese]. Minas Gerais (BR): Universidade Federal de Viçosa; 2002. 52 p. 
46. Siqueira ENL, Bartelli BF, Nascimento, ART, Nogueira-Ferreira FH. Diversity and nesting substrates of stingless bees (Hymenoptera, Meliponina) in a forest remnant. Psyche: A Journal of Entomology. 2012; 2012:1-9, doi: 10.1155/2012/370895.

47. Sakagami SF, Laroca S. Relative abundance, phenology and flower visits of apid bees in eastern Paraná, southern Brazil (Hymenoptera, Apidae). Kontyü. 1971;39(3):217-230.

48. Knoll FRN, Bego LR, Imperatriz-Fonseca VL. As abelhas em áreas urbanas. Um estudo no campus da Universidade de São Paulo. In: Pirani JR, Cortopassi-Laurino M, editores. Flores e Abelhas em São Paulo. São Paulo: Edusp/FAPESP; 1993. p. 31-42.

49. Carvalho CAL, Marchini LC. Abundância de ninhos de Meliponinae (Hymenoptera: Apidae) em biótopo urbano no município de Piracicaba-SP. Rev Agricultura. 1999; (1):35-44, doi: 10.37856/bja.v74i1.1221.

50. Proní EA. Biodiversidade de abelhas indígenas sem ferrão (Hymenoptera: Apidae: Meliponinae) na bacia do rio Tibagi, Estado do Paraná, Brasil. Arq Ciênc Vet Zool da UNIPAR. 2000;3(2):145-150.

51. Taura HM, Laroca S. A associação de abelhas silvestres de um biótopo urbano de Curitiba (Brasil), com comparações espaço-temporais: abundância relativa, fenologia, diversidade e explotação de recursos (Hymenoptera, Apoidea). Acta Biol Paranaense. 2001;30(1-4):35-137.

52. Albuquerque P, Ferreira RDG, Rêgo M, dos Santos CS, de Brito C. Levantamento da fauna de abelhas silvestres (Hymenoptera, Apoidea) na região da" Baixada Maranhense": Vitória do Mearim, MA, Brasil. Acta Amazonica. 2001 31(3):419-419, doi: 10.1590/1809-43922001313430.

53. Sousa LA, Pereira TO, Prezoto F, Faria-Mucci GM. Nest foundation and diversity of Meliponini (Hymenoptera, Apidae) in an urban area of the municipality of Juiz de Fora, MG, Brazil. Bioscience J. 2002;18(2):59-65.

54. Antonini Y, Martins RP. The flowering-visiting bees at the ecological station of the Universidade Federal de Minas Gerais, Belo Horizonte, MG, Brazil. Neotr Entomol. 2003;32(4):565-575, doi: 10.1590/S1519566X2003000400006.

55. Toledo VDAAD, Fritzen AEDT, Neves CA, Ruvolo-Takasusuki, MCC, Sofia SH, Terada Y. Plants and pollinating bees in Maringá, State of Paraná, Brazil. Braz Arch Biol Technol. 2003;46(4):705-710, doi: 10.1590/S1516-89132003000400025.

56. Locatelli de Souza EM. Fenologia e relações abelhas/plantas em uma comunidade de Mata Serrana (Brejo de Altitude) no Nordeste do Brasil [tese]. Pernambuco (BR): Universidade Federal de Pernambuco; 2003. $159 \mathrm{p}$.

57. Anacleto DDA, Marchini LC. Análise faunística de abelhas (Hymenoptera, Apoidea) coletadas no cerrado do estado de São Paulo. Acta Scientiarum. Biol sci. 2005;27(3):277-284, doi: 10.4025/actascibiolsci.v27i3.1315.

58. Viana BF, Kleinert ADMP. A community of flower-visiting bees (Hymenoptera: Apoidea) in the coastal sand dunes of northeastern Brazil. Biota Neotr. 2005;5(2):79-91, doi: 10.1590/S167606032005000300005 .

59. d'Ávila M, Marchini LC. Análise faunística de himenópteros visitantes florais em fragmento de cerradão em Itirapina, SP. Ciênc Florestal. 2008;18(2):271-279, doi: 10.5902/19805098465.

60. Alvarenga PEF. Levantamento da fauna de abelhas (Hymenoptera, Apidae, Meliponina) na mata Santa Tereza, estação ecológica de Ribeirão Preto - SP, e limitantes da densidade de seus ninhos [dissertação]. São Paulo (BR): Universidade de São Paulo; 2008.98 p.

61. Santiago LR, Brito RM, Muniz TMVL, Oliveira FFD, Francisco FDO. A fauna apícola do Parque Municipal da Cachoeirinha (Iporá, GO). Biota Neotr. 2009;9(3):393-397, doi: 10.1590/S167606032009000300034.

62. Mateus S, Pereira UCR, Cabette HSR, Zucchi R. Locais de nidificação das abelhas nativas sem ferrão (Hymenoptera, Apidae, Meliponinae) do Parque Municipal do Bacaba, Nova Xavantina-MT. Mensagem Doce. 2009;1:60-62.

63. Gruchowski-W FC, Iantas J, Maciel L, Holdefer DR. Inventário da fauna de abelhas (Hymenoptera: Apoidea) com a utilização de armadilha atrativa e interceptadora de voo em flora típica sul paranaense. Biodiversidade Pampeana. 2010;8(1):25-31.

64. Cure-Hakim JR, Laroca S. A comunidade de abelhas silvestres (Anthophila) do Parque da Cidade (Curitiba, Brasil): diversidade, abundância relativa, fenologia e recursos tróficos. Acta Biol Paranaense. 2010;39(3-4):111-181.

65. Steiner J, Zillikens A, Kamke R, Feja EP, de Barcellos Falkenberg D. Bees and melittophilous plants of secondary Atlantic forest habitats at Santa Catarina Island, southern Brazil. Oecol australis. 2010; 14(1):16-39, doi: 10.4257/oeco.2010.1401.01.

66. Pioker-Hara FC. Determinantes da densidade e distribuição de ninhos e diversidade de espécies de meliponíneos (Apidae, Meliponini) em áreas de cerrado de Itirapina, SP [tese]. São Paulo (BR): Universidade de São Paulo; 2011. 220 p. 
67. Santos JF. Fenologia da floração e rede de interação entre abelhas e plantas em uma área em processo de restauração florestal localizada em Araras-SP [dissertação]. São Paulo (BR): Universidade Federal de São Carlos; 2012. $61 \mathrm{p}$.

68. Martins AC, Gonçalves RB, Melo GAR. Changes in wild bee fauna of a grassland in Brazil reveal negative effects associated with growing urbanization during the last 40 years. Zoologia. 2013;30(2):157176, doi: 10.1590/S1984-46702013000200006.

69. Aidar IF, Santos AOR, Bartelli BF, Martins GA, Nogueira-Ferreira FH. Nesting ecology of stingless bees (Hymenoptera, Meliponina) in urban areas: the importance of afforestation. Bioscience $\mathrm{J}$. 2013;29(5):1360-1368, doi: 10.5061/dryad.k54hk.

70. Werneck HA, Faria-Mucci GM. Abelhas sem ferrão (Hymenoptera: Apidae, Meliponini) da Estação Ecológica de Água Limpa, Cataguases-MG, Brasil. EntomoBrasilis. 2014;7(2):164-166, doi: 10.12741/ebrasilis.v7i2.408.

71. Sousa VC. Riqueza, abundância relativa e densidade de ninhos de meliponíneos (Apidae, Meliponini) em duas áreas de estágios sucessionais distintos de vegetação do Parque Estadual das Fontes do Ipiranga, São

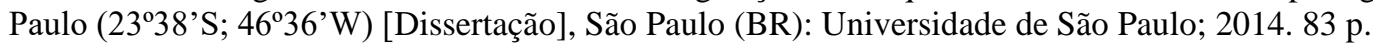

72. Pereira SAN. Levantamento da fauna de abelhas no município de Monte Carmelo-MG. Rev GeTeC. 2015;4(7):11-24.

73. Viera KM (2015). Vespas em ninhos armadilha e nidificação de meliponíneos em um fragmento urbano de mata atlântica, MG [dissertação]. Minas Gerais (BR): Universidade Federal de Juiz de Fora; 2015. 68 p.

74. Sorreque MHC. Interação entre abelhas e flora em remanescentes de cerrado nos municípios de Campo Mourão e Tuneiras do Oeste - Paraná [monografia]. Paraná (BR): Universidade Tecnológica Federal do Paraná; 2016.37 p.

75. Possamai BT, Dec E, Mouga, DMDS. Bee community and trophic resources in Joinville, Santa Catarina. Acta Biol Catarinense. 2017;4(1):29-41, doi: 10.21726/abc.v4i1.360.

76. Sobreiro AI. Influência da regeneração florestal na comunidade de abelhas da Mata Atlântica e Cerrado, Brasil [tese]. Mato Grosso do Sul (BR): Universidade Federal da Grande Dourados; 2018. 103 p.

77. Silva SO. Hortas urbanas e abelhas nativas: um estudo de caso em Foz do Iguaçu - PR [monografia]. Paraná (BR): Universidade Federal da Integração Latino-America; 2019. 42 p.

78. Dantas e Silva M, Ferreira MS, Ferreira, MS. Espécies de abelhas sem ferrão (Apidae: Meliponini) em um fragmento urbano de Mata Atlântica secundária no Recôncavo da Bahia, Brasil. Magistra. 2019;30:189-198. 